\title{
Técnica de transfixión para el tratamiento del glaucoma secundario a bloqueo pupilar por aceite de silicón refractario a iridotomía con láser Nd-YAG
}

\author{
Transfixion technique for the treatment of silicone oil pupillary block glaucoma refractory \\ to Nd-YAG iridotomy
}

\author{
Sergio E. Hernández-Da Mota ${ }^{1,2}$, Rafael Castañeda-Diez ${ }^{3}$ y María Teresa Zavala-Martínez ${ }^{1,2 *}$ \\ ${ }^{1}$ Facultad de Medicina, Universidad Michoacana de San Nicolás de Hidalgo, Morelia, Michoacán; ${ }^{2}$ Unidad Oftalmológica, Clínica David; ${ }^{3}$ Servicio \\ de Glaucoma, Hospital Dr. Luis Sánchez Bunles, Asociación Para Evitar la Ceguera en México (APEC), Ciudad de México, México
}

\section{Resumen}

Antecedentes: El bloqueo pupilar secundario a aceite de silicón en pacientes vitrectomizados es una condición frecuente que normalmente se trata con apertura de la iridectomía con láser Nd-YAG o con el retiro del aceite de silicón. Caso clínico: Se describe un caso de glaucoma secundario a bloqueo pupilar por aceite de silicón tratado satisfactoriamente con técnica de transfixión con aguja de calibre 30. Realizamos una cirugía de vitrectomía sin complicaciones para el tratamiento de un desprendimiento de retina con vitreorretinopatía proliferativa, que incluyó lensectomía, iridectomía periférica inferior e inyección de aceite de silicón. Después de la cirugía, la iridectomía se ocluyó con tejido fibroso y la presión intraocular se incrementó a $50 \mathrm{mmHg}$. Después de que una iridotomía inicial con láser Nd-YAG no tuvo éxito, pasamos una aguja de calibre 30 a través del limbo esclerocorneal y cortamos el tejido fibroso que bloqueaba la iridectomía. Este procedimiento restauró el flujo de humor acuoso a través de la iridectomía, desplazando la burbuja de aceite de silicón a la cavidad vítrea, y la presión intraocular descendió a valores normales. Conclusiones: La técnica de transfixión con aguja de calibre 30 puede ser una alternativa efectiva, simple y de bajo costo para el tratamiento del bloqueo pupilar con aceite de silicón en pacientes con afaquia.

PALABRAS CLAVE: Transfixión. Bloqueo pupilar. Aceite de silicón.

\begin{abstract}
Background: Pupillary block induced by silicon oil in vitrectomized patients is a common condition usually treated with Nd-YAG laser iridotomy or with surgical removal of silicon oil. Case report: A case of silicone oil pupillary block glaucoma successfully treated with a $30 \mathrm{G}$ needle transfixion technique is described. We performed a non-complicated vitrectomy surgery for retinal detachment with proliferative vitreoretinopathy that included lensectomy, inferior peripheral iridectomy, and silicone oil injection. After surgery, the iridectomy became occluded with fibrous tissue and the intraocular pressure raised to $50 \mathrm{mmHg}$. After an initial Nd-YAG iridotomy was unsuccessful, we passed a $30 \mathrm{Ga}$ needle through the sclerocorneal limbus and cut the fibrous tissue that blocked the iridectomy. This procedure restored the aqueous humor flow through the iridectomy, pushed back the silicone oil bubble into the vitreous cavity and lowered the intraocular pressure to normal levels. Conclusions: $30 \mathrm{Ga}$ needle transfixion technique could be an effective, low cost, simple alternative for the treatment of silicone oil pupillary block in aphakic patients.
\end{abstract}

KEY WORDS: Transfixion. Pupillary block. Silicon oil.

\author{
Correspondencia: \\ *María Teresa Zavala Martínez \\ Avda. México, 1020 \\ Fracc. Américas Britania \\ C.P. 58270, Morelia, Mich., México \\ E-mail: teisy_298@hotmail.com
}

Fecha de recepción: 19-04-2018

Fecha de aceptación: 28-04-2018

DOI: $10.24875 / C I R U .18000386$
Cir Cir. 2019;87:215-218

Contents available at PubMed www.cirugiaycirujanos.com 


\section{Introducción}

El glaucoma secundario a bloqueo pupilar en ojos llenos de aceite de silicón es una complicación en algunos pacientes que han sido sometidos a cirugía vitreorretiniana $^{1,2}$. Esto ocurre por la presencia de un bloqueo de la iridectomía inferior en muchos casos. El manejo de esta condición incluye la iridotomía con láser Nd-YAG en ojos con afaquia, la realización de cirugía para retirar el aceite de silicón y la reapertura de la iridectomía ocluida con la punta del vitrector. Estos procedimientos a veces no son efectivos, pueden llegar a ser costosos y demandan mayor tiempo, sobre todo al tener que reintervenir en quirófano al paciente. El propósito de este reporte de caso es describir un procedimiento ambulatorio, realizado en el consultorio, para tratar esta condición en ojos con afaquia y presencia de aceite de silicón.

\section{Reporte de caso}

Una mujer de 50 años con antecedente de desprendimiento de retina con vitreorretinopatía proliferativa en su ojo derecho, que recientemente había sido sometida a vitrectomía, lensectomía, cerclaje escleral e inyección de 5 cc de aceite de silicón «5000 Centistokes", reportó intenso dolor y disminución de la agudeza visual del mismo ojo de 4 días de evolución. También reportó que se le había realizado una iridotomía con láser Nd-YAG para el tratamiento de su condición, sin presentar mejoría en la sintomatología. Se tomó una presión intraocular de $50 \mathrm{mmHg}$ y se observó aceite de silicón llenando toda la cámara anterior. En el lugar de la iridotomía se apreció la presencia de tejido fibroso con acúmulos de pigmento bloqueando la iridectomía inferior (Fig. 1).

\section{Procedimiento quirúrgico}

Primeramente se aplicó a la paciente anestesia retrobulbar con 3.5 cc de lidocaína al $2 \%$ y bupivacaína al $0.75 \%$ en una mezcla de 50:50. Se empleó una técnica estéril que incluyó solución de povidona yodada para esterilizar los párpados y la superficie conjuntival, guantes estériles, cubrebocas y un blefarostato. A continuación, se colocó a la paciente en la lámpara de hendidura.

Se pasó una aguja de calibre 30 montada en una jeringa de $1 \mathrm{cc}$ a través del limbo esclerocorneal en el meridiano de las 5, aproximadamente, y a través

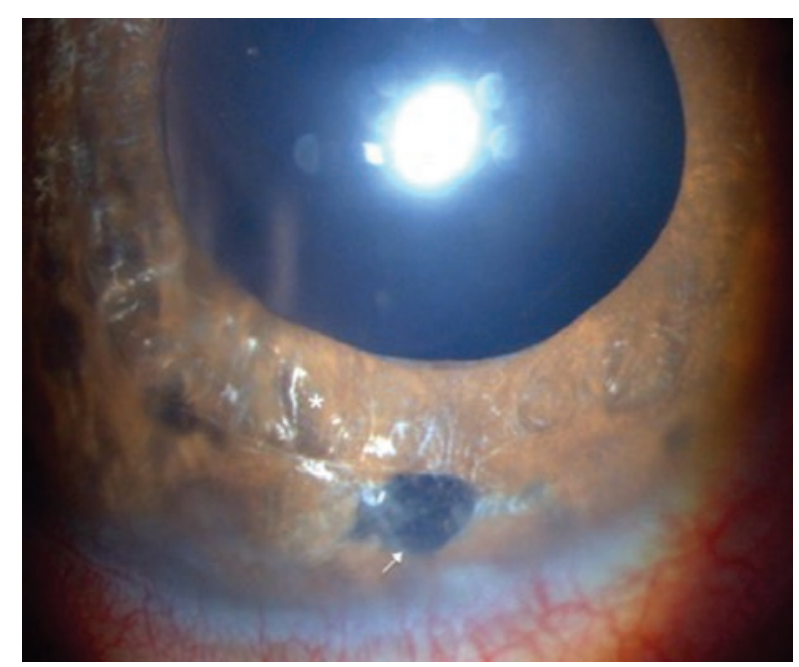

Figura 1. Imagen que muestra la cámara anterior llena con aceite de silicón (el asterisco indica un reflejo en el iris debido a la presencia del aceite de silicón que llena la cámara anterior) y la iridectomía bloqueada con tejido fibroso y acúmulos de pigmento (flecha).

de la cámara anterior y del sitio de la iridectomía perforando el tejido fibroso que la bloqueaba. Después se sacó la aguja y se aplicó presión durante algunos segundos con la punta de un aplicador de algodón sobre el sitio de entrada al limbo, para evitar filtraciones y descompresiones bruscas al final del procedimiento. Esta maniobra restauró el flujo de humor acuoso a través de la iridectomía, desplazando la burbuja de aceite de silicón de la cámara anterior hacia la cavidad vítrea. Treinta minutos después del procedimiento, la presión intraocular había disminuido a $17 \mathrm{mmHg}$ (Fig. 2). Se prescribió medicación tópica a base de prednisolona y gatifloxacino por 7 días para tratar la inflamación posoperatoria y como manejo profiláctico ante infecciones.

Un mes después del procedimiento, la iridectomía se apreció permeable y la presión intraocular era de $18 \mathrm{mmHg}$. Se resume la técnica en el esquema de la figura 3.

\section{Discusión}

En el pasado, una complicación posoperatoria temprana en ojos con afaquia llenos de aceite de silicón era la hipertensión ocular, y para prevenirla se recomendaba la creación de una iridectomía periférica inferior. La iridectomía, realizada al final de la cirugía, pero antes de la inyección de aceite de silicón, debía ser lo suficientemente grande para prevenir la obstrucción por células inflamatorias ${ }^{1}$.

Sin embargo, el glaucoma por bloqueo pupilar sigue ocurriendo en ojos con afaquia, en general 


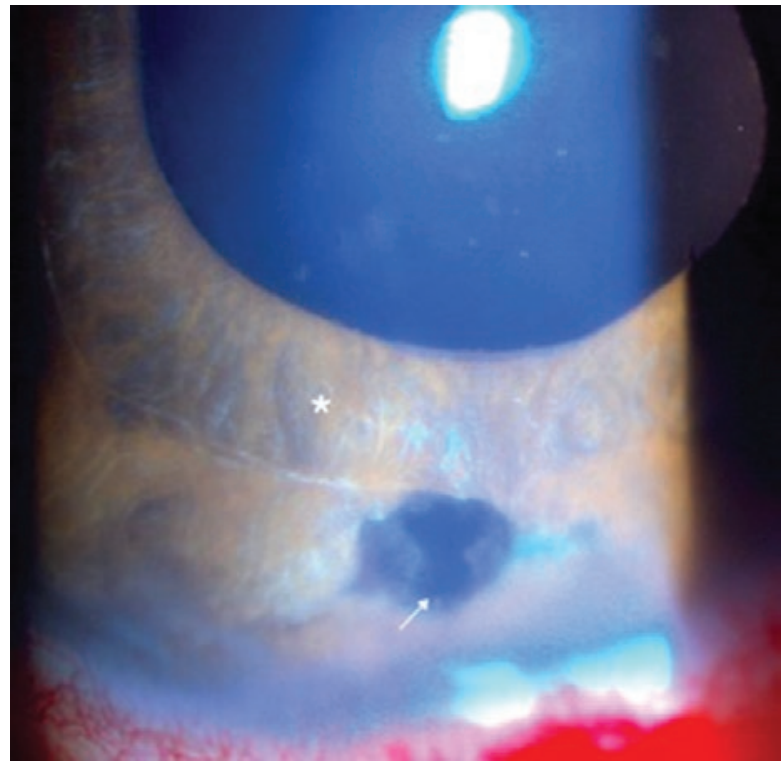

Figura 2. Imagen del segmento anterior 30 minutos después del procedimiento de transfixión con aguja. Hay una abertura hecha con la aguja en el tejido fibroso que bloqueaba la iridectomía que restableció el flujo de humor acuoso a través de esta (flecha). El reflejo del iris por la presencia de aceite de silicón en la cámara anterior ha desaparecido (asterisco) porque la burbuja fue desplazada hacia la cavidad vítrea por el humor acuoso.
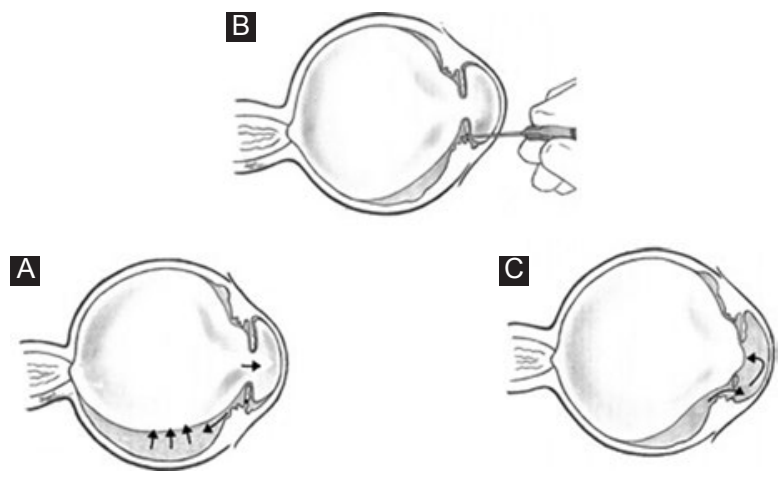

Figura 3. Esquema que muestra la iridectomía bloqueada y la burbuja de aceite de silicón dentro de la cámara anterior (A), la aguja de calibre 30 usada para la transfixión cortando el tejido fibroso que bloqueaba la iridectomía (B), y el humor acuoso fluyendo a través de la iridotomía restaurada y la burbuja de aceite de silicón desplazada hacia la cavidad vítrea (C).

durante el posoperatorio temprano, cuando la iridectomía periférica no está funcionando. Esta complicación tiene una incidencia reportada superior al 33\%2. Cuando la iridectomía periférica no es funcional, el humor acuoso se acumula detrás del iris y desplaza la burbuja de aceite de silicón a través de la pupila. El bloqueo pupilar es el resultado de la progresión de este fenómeno.

Después de la operación, la iridectomía inferior puede ocluirse con depósitos de fibrina, sangre 0 remanentes de la bolsa capsular que permanecen en el ojo e inducen la proliferación al sitio de iridectomía, dando como resultado unos valores elevados de presión intraocular. En estos casos, el control de la inflamación con esteroides, láser $\mathrm{Nd}$ YAG u otra cirugía puede reabrir la iridectomía ${ }^{3}$. Si la causa del bloqueo es por fibrina o por un coágulo, la inyección de factor activador del plasminógeno sobre el tejido dentro de la cámara anterior ha resultado exitosa ${ }^{4}$.

Además, la iridotomía con láser Nd-YAG en casos de glaucoma secundario a bloqueo pupilar por aceite de silicón fracasa en un 15.4 y un $38.5 \%$ a corto y largo plazo, respectivamente, según se ha reportado ${ }^{5}$. Si una iridotomía con láser Nd-YAG inicial o reoperación falla para reabrir la iridectomía, o esta se cierra de nuevo al poco tiempo, el cirujano puede realizar este procedimiento varias veces hasta que permanezca abierta. Sin embargo, esto puede llegar a ser muy costoso y causar cierto desagrado, molestia o franco dolor al paciente. Numerosas cirugías también pueden llevar a otras complicaciones, y es mayor el tiempo perdido.

Las técnicas de transfixión para el tratamiento del glaucoma agudo fueron descritas por Fuchs y Arruga generalmente para casos de sinequias posteriores e iris bomba. La descripción de su método, que incluía el uso de un cuchillete largo para cirugía de catarata que se pasaba a través del limbo esclerocorneal y el iris, algunas veces conducía a una descompresión brusca de la cámara anterior en el momento de sacarlo, y a otras complicaciones tales como hemorragia supracoroidea ${ }^{6}$.

No observamos esta descompresión con la técnica que describimos debido a que la herida hecha con una aguja de calibre 30 es muy pequeña y autosellable, y además aplicamos presión con un aplicador de algodón al final del procedimiento.

Este método es un procedimiento fácil, eficiente y sencillo, que se realiza en el consultorio, para el manejo del glaucoma secundario a bloqueo pupilar por aceite de silicón, y que no tiene todas las desventajas de los tratamientos convencionales ya mencionados para tratar esta complicación que se presenta comúnmente en ojos con afaquia llenos de aceite de silicón.

\section{Agradecimientos}

Los autores desean agradecer a Fernando Zúñiga los dibujos esquemáticos de la técnica. 


\section{Financiamiento}

El presente artículo no tuvo ninguna fuente de financiamiento.

\section{Conflicto de intereses}

No hay conflicto de intereses por parte de los autores.

\section{Responsabilidades éticas}

Protección de personas y animales. Los autores declaran que para esta investigación no se han realizado experimentos en seres humanos ni en animales.
Confidencialidad de los datos. Los autores declaran que en este artículo no aparecen datos de pacientes.

Derecho a la privacidad y consentimiento informado. Los autores declaran que en este artículo no aparecen datos de pacientes.

\section{Bibliografía}

1. Ando F. Intraocular hypertension resulting from pupillary block by silicon oil. Am J Ophthalmic. 1985;99:87-8.

2. Madreperla SA, McCuen BW II. Inferior peripheral iridectomy in patients receiving silicone oil. Retina.1995;15:87-90.

3. Bartov E, Huna R, Ashkenazi I, Melamed S, Gutman I, Naveh N, et al. Identification, prevention and treatment of silicone oil pupillary block after an inferior iridectomy. Am J Ophthalmol. 1991;111:501-4.

4. MacCumber MW, McCuen BW II, Toth CA, Ferrone PJ, Jaffe GJ. Tissue plasminogen activator for preserving inferior peripheral iridectomy patency in eyes with silicone oil. Ophthalmology. 1996;103:269-73.

5. Zalta AH, Boyle NS, Zalta AK. Silicone oil pupillary block: an exception to combined argon-Nd: YAG laser iridotomy success in angle-closure glaucoma. Arch Ophthalmol. 2007;125:883-8.

6. Arruga $H$. Ocular surgery. $5^{\text {th }}$ ed. Barcelona: Salvat; 1963. 